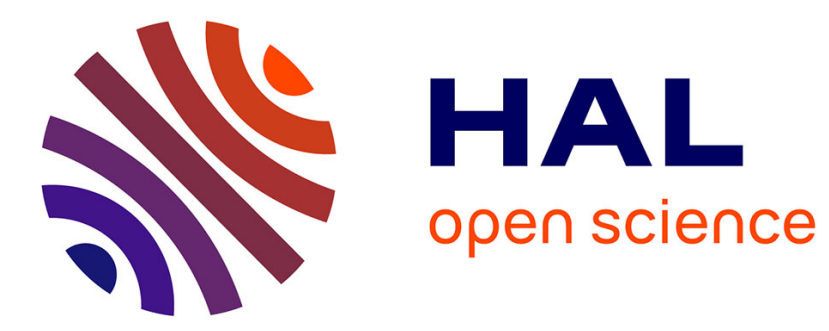

\title{
Well-controlled foam-based solid coatings
}

Aymeric Mouquet, Yacine Khidas, Tamar Saison, Jean-Yvon Faou, Olivier

Pitois

\section{To cite this version:}

Aymeric Mouquet, Yacine Khidas, Tamar Saison, Jean-Yvon Faou, Olivier Pitois. Well-controlled foam-based solid coatings. Soft Matter, 2019, 15 (25), pp.5084-5093. 10.1039/c9sm00786e . hal02930194

\section{HAL Id: hal-02930194 \\ https://hal-enpc.archives-ouvertes.fr/hal-02930194}

Submitted on 4 Sep 2020

HAL is a multi-disciplinary open access archive for the deposit and dissemination of scientific research documents, whether they are published or not. The documents may come from teaching and research institutions in France or abroad, or from public or private research centers.
L'archive ouverte pluridisciplinaire HAL, est destinée au dépôt et à la diffusion de documents scientifiques de niveau recherche, publiés ou non, émanant des établissements d'enseignement et de recherche français ou étrangers, des laboratoires publics ou privés. 


\title{
Well-controlled foam-based solid coatings
}

\author{
A. Mouquet ${ }^{1,3}$, Y. Khidas ${ }^{2}$, T. Saison ${ }^{3}$, J.-Y. Faou ${ }^{3}$, and O. Pitois ${ }^{1, *}$ \\ ${ }^{1}$ Université Paris Est, Laboratoire Navier, UMR 8205 CNRS - École des Ponts ParisTech - IFSTTAR \\ cité Descartes, 2 allée Kepler, 77420 Champs-sur-Marne, France. \\ aymeric.mouquet@gmail.com ; olivier.pitois@ifsttar.fr \\ ${ }^{2}$ Université Paris Est, Laboratoire Navier, UMR 8205 CNRS - École des Ponts ParisTech - IFSTTAR \\ 5 bd Descartes, 77454 Marne-la-Vallée Cedex 2, France. \\ vacine.khidas@u-pem.fr \\ ${ }^{3}$ Saint-Gobain Research Paris, 39 Quai Lucien Lefranc, 93300 Aubervilliers, France \\ tamar.saison@saint-gobain.com ; jean-yvon.faou@saint-gobain.com
}

\begin{abstract}
Foam-based solid coatings appear to be a simple solution for giving new properties to solid surfaces. An efficient method is presented for producing open-cell foam coatings having tunable pore radius distribution (i.e. monodisperse within the range 100-1000 $\mu \mathrm{m}$, bidisperse or fully polydisperse), tunable thickness, and tunable bulk and surface porosities. This is achieved by mixing precursor aqueous foam and particle suspension (here a micrometer-sized polyurethane dispersion), and by spreading with a nozzle the resulting particle-loaded foam on the solid surface to be coated. It is shown that bubble size distribution of the precursor foam can be preserved in the final solid coating. This this highlighted by using monodisperse aqueous foam, for which coatings showed polycrystalline structure, as well as bidisperse or fully polydisperse foams. As a major advantage of our method, bubble size and solid volume fraction are shown to be independent parameters allowing for the size of the microstructural elements to be tuned easily, so are the expected functional properties of the coating. Results obtained with the studied polyurethane dispersion are expected to be reproduced with other dispersions.
\end{abstract}

\section{Introduction}

Chemical functionalization is widely used for tuning surface interactions between nano-scaled or macroscopic objects. On the other hand, modification of surface geometry has proven to provide enhanced surface properties. For example textured surfaces are known to enhance hydrophobic properties [1]. Bio-inspired coatings with fibrillar structures provide strongly enhanced adhesion compared with the uncoated flat surface of same material [2]. In fact, coating's morphology could be 
used as a powerful way for tuning several crucial physical properties of solid surfaces, such as mechanics [3] or optics [4] for example. Achieving such morphology control over large scales is still challenging and, to this regard, foam templating appears as an interesting method: liquid foams can be produced in significant amount, then deposited on the substrate and hold as a coating thanks to their complex rheological properties, and finally solidified, hopefully by keeping the foam tridimensional structure. Numerous solidification processes can be used, depending on foaming solution [5]: gelation, polymerization, sintering, ... Obviously, good adhesion (e.g. van der Waals attraction) between the two materials (coating/substrate) is necessary. Foam coating templating is not expected to cancel this requirement: the solid foam coating will adhere to the substrate if the material to be foamed itself adheres to the substrate.

It is to say that best control of foam structure was obtained by using microfluidic technics, where each one of the foam bubbles is produced through a unique orifice (for example [6], [7]). On demand tuning of the orifice allows even for pore size gradients to be created [8]. However, production rates using such microfluidic devices are intrinsically low. For coating purpose, this would involve additive manufacturing or coating small substrates. Recent study on coating process using microfluidics showed that controlling the resulting solid structure remains challenging [9] due to significant evolution of the foam structure before solidification.

In the following we present a simple method for producing solid coatings with well-controlled morphology. Coatings are built up by a two-steps method, where aqueous precursor foam is first produced and collected in a reservoir, then mixed with a particle suspension (here micrometer-sized polyurethane dispersion) and spread at significant velocity on the surface to be coated. The fresh coating is rapidly solidified by partial evaporation of the suspending water while keeping the main features of the morphology that has been set during the spreading step. Such a method is proven for allowing simple tuning of all the geometrical features of the coating, i.e. the thickness, the pore size distribution, and the characteristic sizes of the structural elements. In the following the method used to coat solid surfaces with thin foam layers is presented. Then results in terms of morphological control of those coatings are presented and discussed.

\section{Materials and methods}

\subsection{Materials}


A polyurethane dispersion (PUD) is used. It is made by mixing an aqueous suspension of $1 \mu m$ surfactant-stabilized polyurethane particles with a cross-linker. The "consolidation" of the PU matrix with a cross-linker (activated by curing) is a necessary step for further applications, especially those involving mechanics, but it is not necessary to make the solid foam coating. The average particle volume fraction is $\varphi_{P U D}^{0} \approx 55 \%$ (45\% water). The density and the shear viscosity of the PUD are $1100 \mathrm{~kg} / \mathrm{m}^{3}$ and $\mu_{0} \approx$ 1 Pa.s (measured at low shear rate, i.e. $1 \mathrm{~s}^{-1}$ ) respectively. As explained in more details in the following, the stability of the fresh PUD foam is crucial, so the surfactant used for foaming had to be chosen appropriately. Indeed, polyurethane particles in aqueous suspensions are well known to be charged (due to the presence of ionic internal emulsifiers groups on the polymer chain for the need of inversion phase process [10]), which may lead to brutal foam collapse depending of the foam's surfactant. Preliminary stability tests led us to prepare foams from Tetradecyltrimethylammonium bromide (TTAB) aqueous solution at a concentration $10 \mathrm{~g} \cdot \mathrm{L}^{-1}$ (approximately tenfold the critical micelle concentration). The surface tension of the foaming solution is $\gamma \approx 0.04 \mathrm{~N} / \mathrm{m}$.

In order to study in detail the morphology of the produced coating, it was necessary to remove them without damage from the substrates. Adhesion of the coating on glass or plastic sheet was found to be too strong, so we turned to silicone coated plastic sheets. Except for the cohesion issue, we did not notice any change in the coating process for the different substrates. Roughness of the used plastic sheets is below $10 \mu \mathrm{m}$ and it is not expected to influence the reported results.

\subsection{Production of the foam coatings}

In broad outline, coatings are produced by mixing precursor aqueous foam with the abovedescribed PUD. The production process can be separated into several steps, as described in Figure 1: (1) production of the precursor aqueous foam, (2) mixing of the precursor aqueous foam with the PUD and shaping of the resulting PUD fresh foam, and (3) drying of the PUD foam coating. Foam content throughout the whole process is sketched in figure $1 \mathrm{~b}$. Note that curing $\left(10 \mathrm{~min}\right.$ at $\left.150^{\circ} \mathrm{C}\right)$ is performed $12 \mathrm{~h}$ after the drying step in order to fix the coating's structure and to improve the mechanical properties of the PUD. The three steps are described in the following. Note that subsequent steps, such as surface treatment could be considered if required, but those are beyond the scope of the present paper. 

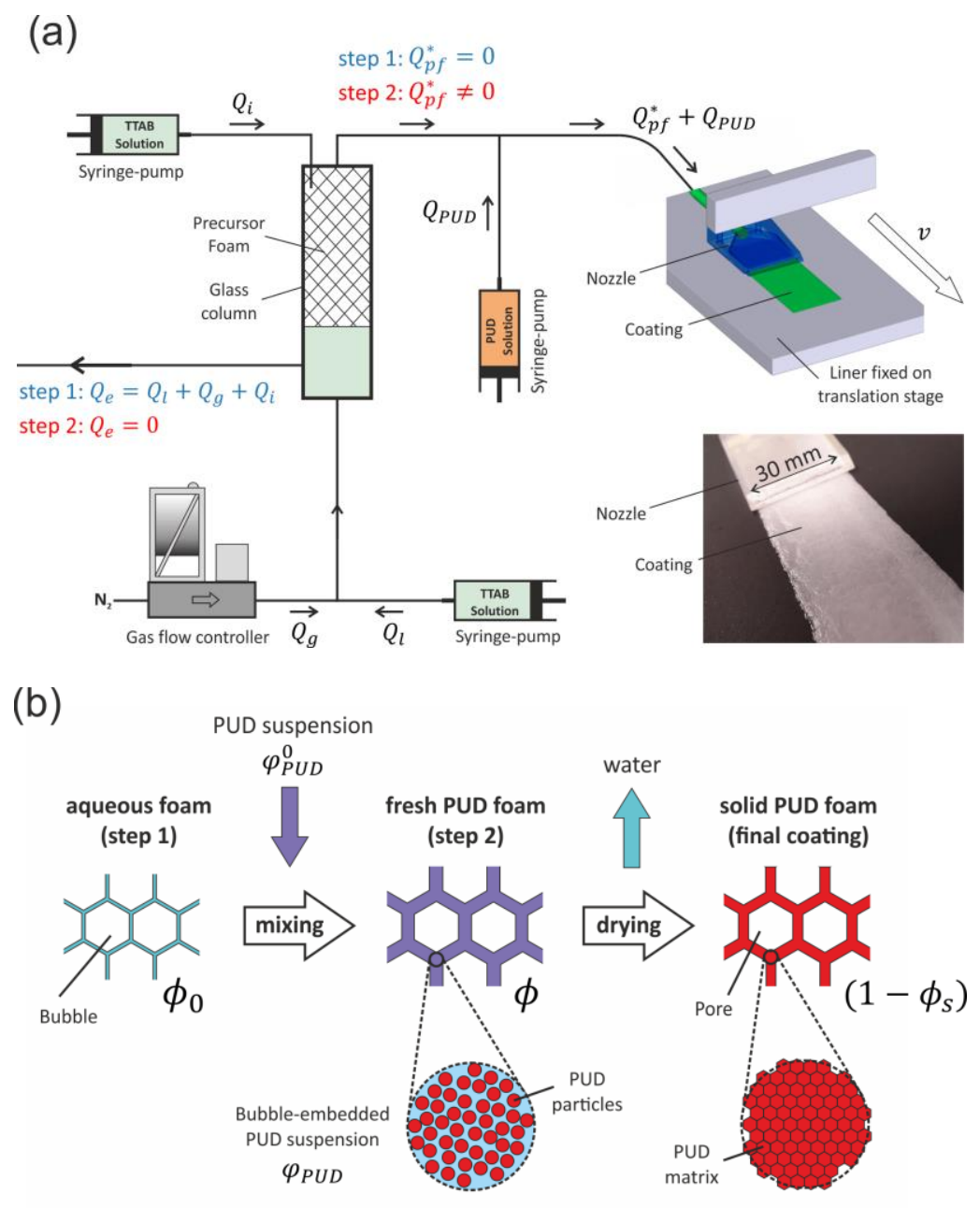

Figure 1: (a) Complete scheme of the foaming device used to produce the fresh PUD foam coatings. The process consists in two consecutive main steps: (step 1) Monodisperse aqueous foam production using microfluidics. The control of the bubble size is made at this step by tuning both gas and liquid volume flow rates, i.e. $Q_{g}$ and $Q_{l}$

respectively. Foaming solution (TTAB) is delivered at flow rate $Q_{i}$ from the top of the column in order to compensate gravity drainage occurring through the foam. (step 2) After production of the precursor foam, the latter is pushed (at flow rate $Q_{p f}^{*}$ ) with the PUD (at flow rate $Q_{P U D}$ ) through a static mixing device and the shaping

of the fresh PUD foam is performed thanks to a nozzle. In some way, the precursor foam is "filled" with the suspension during the mixing process. The proportions of precursor foam/PUD suspension are controlled by tuning their volume flow rates, and these parameters have been shown to set also the final solid volume fraction. The surface to be coated (here a plastic liner) is moved at controlled velocity. The image shows the resulting foam coating deposited by the device on the liner. (b) Sketches of the foam content throughout the whole process. Left: Precursor aqueous foam contains water ( $\phi_{0}$ is the gas volume fraction). Center: the fresh PUD foam contains PUD suspension ( $\phi$ is the gas volume fraction and $\varphi_{P U D}$ is the PUD particle volume fraction in the bubble-embedded PUD suspension. Right: the final solid foam coating contains PUD solid matrix ( $\phi_{s}$ is the solid volume fraction). Note that the solid matrix results from the drying-induced densification of the initially dispersed PUD particles. 


\subsubsection{Step 1: Production of precursor aqueous foam}

Significant work was devoted to the control of the bubble size distribution in the precursor aqueous foam. The latter is generated by pushing both gas (nitrogen) and the foaming liquid (TTAB solution) through a T-junction (see Figure 1a). Gas volume flow rate $Q_{g}$ is set within the range 0.1$10 \mathrm{~mL} \cdot \mathrm{min}^{-1}$ by using a gas mass flow controller. The foaming solution is pushed at volume flow rate $Q_{l}$ thanks to a syringe pump. Tuning the flow rates ratio $Q_{g} / Q_{l}$ through a T-junction with a circular crosssection of diameter $1.6 \mathrm{~mm}$ was found appropriate to produce bubbles with diameters $D_{b}$ ranging between $800 \mu \mathrm{m}$ and few millimeters. In order to obtain smaller bubble sizes, typically from $400 \mu \mathrm{m}$ to $800 \mu \mathrm{m}$, the internal diameter of the T-junction was reduced by inserting a glass capillary with external diameter fitting T-junction's walls. Following the same principle, even smaller bubbles (i.e. $D_{b} \approx 150 \mu \mathrm{m}$ ) have been produced by reducing the cross-section of the glass capillary. This was achieved by using a micropipette puller (Vertical Micropipette Puller P-30 from Sutter Instrument). Note that reducing the bubble size results in a strong decrease of the flow rates' operating range: $Q_{g} \approx 5 \mathrm{~mL} \mathrm{~min}^{-1}$ for the large bubbles, $Q_{g} \approx 0.5 \mathrm{~mL} \cdot \mathrm{min}^{-1}$ for small bubbles.

Generated bubbles are continuously collected into a vertical glass column (see Figure 1) initially filled with foaming solution. The average bubble diameter is measured using a camera focused at the wall of the column. As the bubble generation process requires a significant amount of liquid (i.e. typically $50 \%$ liquid vs $50 \%$ gas), excess liquid is removed from the column thanks to an overflow outlet (as indicated by flow rate $Q_{e}$ in Figure 1). Note that foam ripening turns out to be prevented over the duration of the production step (i.e. the duration of the production process is shorter than the time required for ripening to be observed within our experimental conditions), so monodisperse precursor aqueous foams were obtained. Bidisperse foam coatings were generated by mixing two monodisperse precursor aqueous foams with different bubble sizes. In such a case, a second generation column was used in parallel. Note also that polydisperse foams were made out of $320 \mu \mathrm{m}$ monodisperse bubbles (obtained as described above), by letting time for the coarsening process to act in the foam column. Foam stability was improved during the whole foaming process by pouring foaming solution from the top of the column (see Figure 1a) with small flow rate $Q_{i}$ (typically $0.1 \times Q_{g}$ ). As a result of those imbibition/drainage conditions, constant gas volume fraction $\phi_{0}=0.92 \pm 0.02$ was obtained over the most part of the foam column (i.e. except for the $3-4 \mathrm{~cm}$ at the bottom [11]). Note that a quantitative control of $\phi_{0}$ can be achieved by tuning $Q_{i}$ thanks to predictions from numerical simulations such as those reported in Gorlier et al. [12] for example. 


\subsubsection{Step 2: mixing of the PUD with the precursor foam, and shaping}

After production of the required precursor foam volume, the latter is pushed with the PUD through a static mixer (flow focusing device and classical helical geometries). Such process is described elsewhere (for example [12]-[15]) for various materials to be mixed with the aqueous foam. The gas volume fraction within the resulting PUD foam (and so the solid volume fraction $\phi_{S}$ in the final coating) can be adjusted by tuning the ratio of flow rates for the precursor foam $\left(Q_{p f}\right)$ and the PUD $\left(Q_{P U D}\right)$. Additional tuning parameters can be used if required: the gas volume fraction $\phi_{0}$ and the PUD volume fraction $\varphi_{P U D}^{0}$. Note that due to gas compressibility and moderate pressurization of the aqueous foam upstream of the mixing device, the true delivered volume flow rate $Q_{p f}^{*}$ (equivalent to atmospheric pressure conditions) is a little bit larger than the targeted value $Q_{p f}$. The pressure in the mixing chamber is expected to increase as a function of the flow rate of PUD solution, so in a first approach, we have accounted for this effect by the following relation: $Q_{p f}^{*} / Q_{p f}=1+c Q_{P U D} / Q_{p f}$, where $c$ is a numerical coefficient and the ratio $c Q_{P U D} / Q_{p f}$ represents the excess pressure in the foam column. The parameter $c$ will be determined from the results detailed in the next section.

In the case where two foam production columns are used, pre-mixing of the two precursor foams is performed upstream thanks to dedicated co-flow geometry. Proportions of the mixed aqueous foams are set by the ratio of their respective flows rates.

A dedicated nozzle is fed with the PUD foam from the mixing device at a flow rate $Q_{p f}^{*}+Q_{P U D}$ (i.e. typically $10 \mathrm{~mL} / \mathrm{min}$ ) and it is allowed to slide onto a plastic sheet liner moved at constant velocity thanks to a motorized translation stage (the stage velocity $v$ can be set between $0.1 \mathrm{~mm} \cdot \mathrm{s}^{-1}$ and to 40 $\mathrm{mm} . \mathrm{s}^{-1}$, with a precision of $0.01 \mathrm{~mm} . \mathrm{s}^{-1}$ ). The dimensions of the nozzle are chosen according to the desired film thickness and width: height $h$ between $0.3 \mathrm{~mm}$ and $3 \mathrm{~mm}$, and fixed width $w=30 \mathrm{~mm}$. The thickness of the resulting coating turns out to be tuned by adjusting the PUD foam flow rate and/or the translation stage velocity (this point will be discussed in paragraph 3.4). The typical covering rate is $200 \mathrm{~cm}^{2} / \mathrm{min}$. 


\subsubsection{Step 3: Drying}

The fresh PUD foam coating is solidified thanks to this last step. This is achieved by evaporating the water from the fresh coating in a climatic chamber during $12 \mathrm{~h}$ at $\mathrm{T}=20^{\circ} \mathrm{C}$ and relative humidity $\mathrm{RH}=$ $40 \%$. Note that (1) the structure of the coating appeared to be already set after $30 \mathrm{~min}$ in the chamber (but complete water removal requires more time, i.e. $12 \mathrm{~h}$ ), and (2) $\mathrm{RH}=40 \%$ appeared as a compromise: When $\mathrm{RH}<40 \%$, water evaporates more quickly and PUD particle dragging driven by stronger internal water flows results in foams with denser top surface, i.e. inhomogeneous foam coatings across their thickness. Additional effects were observed: the foam started to solidify locally, which means that bubbles were not allowed to rearrange for adapting the significant shrinkage induced by water loss, leading sometimes to fractures in the film, as already observed in [9]. On the opposite, when RH $>40 \%$, the solidifying process took longer time, promoting therefore aging processes such as drainage or coarsening. This often resulted in density gradient across the sample thickness.

\subsection{Morphology characterization}

Several samples of size close to $1 \mathrm{~cm} \times 1 \mathrm{~cm}$ are cut within the dried foam coating and removed from the liner. Note that such removal was possible only with the silicone coated substrates. The sample area $S$ is then measured accurately using image processing from a magnified picture of the sample as observed from above (optical microscopy - Zeiss Stereo Discovery V.8). The same method is used to measure the sample's thickness $e$. The mass $m$ is measured with a precision scale and the corresponding density $m /(S e)$ is determined. The solid volume fraction $\phi_{s}=m /\left(S e \rho_{m}\right)$ is then determined from the measured density of the dried PUD, i.e. $\rho_{m}=1100 \mathrm{~kg} \cdot \mathrm{m}^{-3}$. Scanning Electron Microscopy (SEM - Bruker quanta $400 \mathrm{FEl}$, tungsten filament, Pt coating $2 \mathrm{~nm}$ ) was used in order to assess the size of the structure elements. X-ray tomography (EasyTom RX Solutions) was used to obtain the three dimensional distribution of the linear X-ray attenuation coefficient through the sample. The distribution forms a 3D image which elementary unit is called a voxel. Voxel size was about $5 \mu \mathrm{m}$ in our experiments. Thanks to the difference in X-ray absorption between $\mathrm{PU}$ and air, and resulting contrast on 3D images, it is possible to separate the pore from the polymer and determine the pore size distributions of the sample. The latter is determined from image analysis using the open source software Fiji [16], a distribution of ImageJ [17]. In order to reduce the noise in the tomography images, we apply a 3D closing filter with a 4 voxelradius ball element provided by the MorphoLibJ plugin [18]. This filter is a common morphological 
dilatation followed by morphological erosion. This procedure removes structures smaller than the structuring element and consequently connects bright structures initially separated by thin dark voxels. Using the Otsu method [19], then the stack of images is binarized using an automatic threshold (i.e. calculated for each image) in order to restrain the mean gray level fluctuations related to local sample density variations. At this stage, we have a binary image separating PU and air in the sample. In order to isolate the different bubbles, the 3D Distance Transform Watershed from the same MorphoLibJ plugin is used, which is a combination of a 3D distance transform of the 3D binary image and a 3D watershed algorithm. 3D Object Counter gives the volume $V_{p}$ for each pore, and therefore we characterize the pore size distribution of our samples from an equivalent diameter under the assumption of spherical pore shape $\left(D_{p}=\sqrt[3]{6 V_{p} / \pi}\right)$. One should note that during the watershed algorithm, no difference is made between gas in our sample and the gas outside. Thus, in order to prevent the selection of the latter volume with the algorithm, we cut the top (and bottom) surface roughness. Note that this process results in artificially reducing the measured pore diameter for a small proportion of the surface pores, but the maximum error made for those pores has been estimated to be close to $10 \%$, which falls within the range of the intrinsic pore size distribution.

\section{Results and discussion}

Using the foam production device presented in Fig. 1a and settings previously explained, several foam coatings have been made with a large range of values for the pore diameter $D_{p}$, the solid volume fraction $\phi_{S}$ or coating thickness $e$. In the following the morphological features of those samples are presented and discussed.

\subsection{Control of the pore size distribution}

Figure 2 shows typical results for the pore size $D_{p}$ in solid foam coatings, as measured from the corresponding tomography images. Coatings made with monodisperse aqueous foam turn out to be characterized by a small relative standard deviation for the pore sizes: $\Delta D_{p} / D_{p} \approx 3 \%$. This indicates that neither coalescence (i.e. bubble merging) nor ripening (i.e. coarsening due to gas transfer from small to large bubbles) did occur significantly during the drying process. We stress that ripening of monodisperse aqueous foam exhibits a so-called transient regime, where coarsening initiates first at topological defects before spreading to ordered areas. Such process is known to delay significantly the effects of ripening at 
the scale of the sample [11]. The ratio $D_{p} / D_{b}$ was measured to be equal to unity within the experimental error bar $\left(\frac{\Delta\left(D_{p} / D_{b}\right)}{D p / D_{b}}=\frac{\Delta D_{b}}{D_{b}}+\frac{\Delta D_{p}}{D p} \approx 10 \%\right)$ within the investigated size range 280-1700 $\mu \mathrm{m}$. We did not observe any trend for this size ratio as a function of $\phi_{s}$, which means that the drying-induced shrinking has no significant effect on $D_{p}$ for a given $D_{b}$ value.

\section{monodisperse}
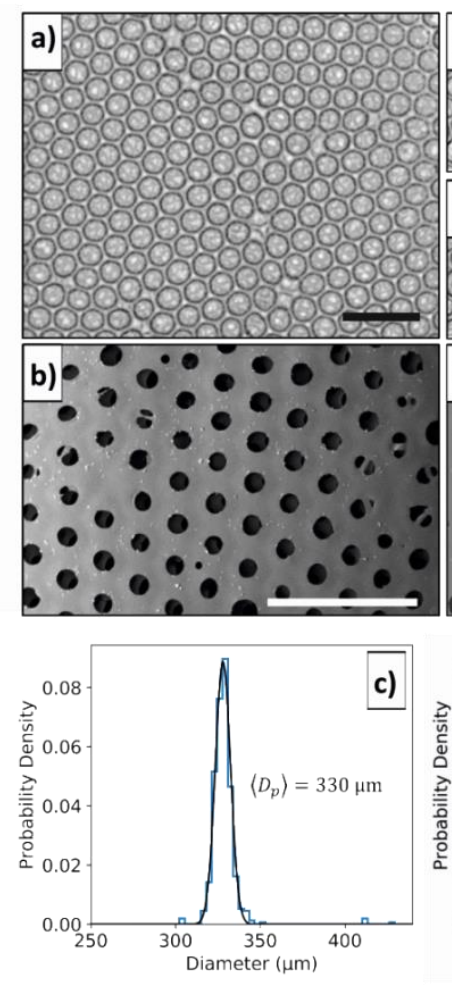

bidisperse
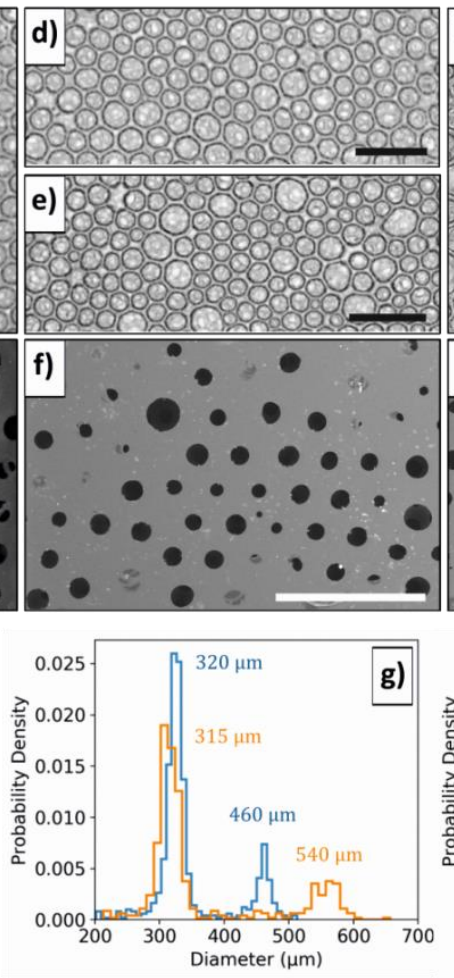

polydisperse
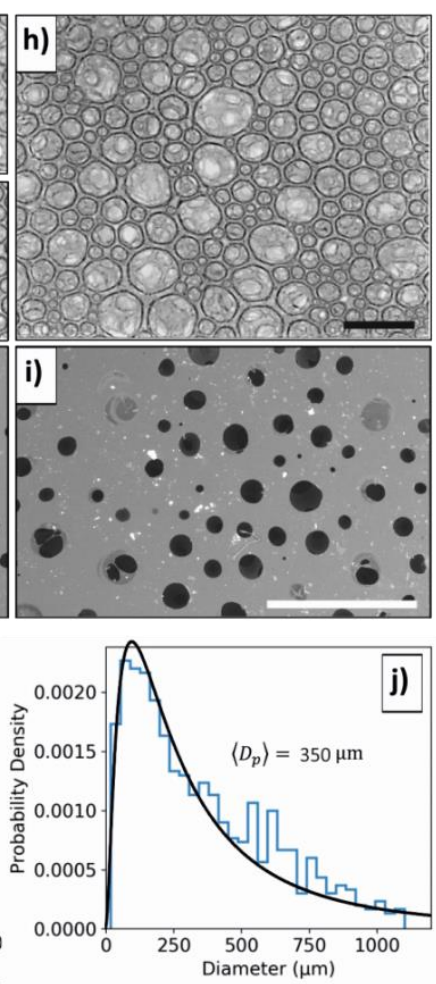

Figure 2: Pore size in foam coatings. Pictures show the top surface of the samples and each scale bar corresponds to $1 \mathrm{~mm}$. (a-c) monodisperse coating (pore diameter: $330 \mu \mathrm{m}$ ): (a) optical microscopy, (b) SEM picture, (c) number weighted pore diameter distribution. The solid line represents a normal distribution with parameters $m_{n}=330$ and $\sigma_{n}=4.8$ (respectively the mean and the standard deviation of the size distribution). (d-g) Bidisperse coatings (number proportions of small/large pores: $80 \% / 20 \%$ ): (d) optical microscopy (pore diameters: $320 \mu \mathrm{m}$ and $460 \mu \mathrm{m}$ ), (e) optical microscopy (pore diameters: $315 \mu \mathrm{m}$ and 540 $\mu \mathrm{m}$ ) (f) SEM picture (pore diameters: $315 \mu \mathrm{m}$ and $540 \mu \mathrm{m}$ ), (g) number weighted pore diameter distributions (320/460 and 315/540 $\mu \mathrm{m}$ ). (h-j) Polydisperse samples (measured average pore diameter: $350 \mu \mathrm{m}$ ): (h) optical microscopy, (i) SEM picture, (j) number weighted pore diameter distribution. The solid line represents a log-normal distribution with parameters $m_{l n}=5.65$ and $\sigma_{l n}=1$ (respectively the mean and the standard deviation of the size's natural logarithm).

For samples made from bidisperse foam characterized by two bubble diameters $D_{b 1}$ and $D_{b 2}>D_{b 1}$, one of the concerns is the coexistence of two Laplace pressures, i.e. $4 \gamma / D_{b 1}$ and $4 \gamma / D_{b 2}$, which is expected to promote efficient ripening. However, as it can be seen in Figure 2, mixing two 
monodisperse foams does not lead to significant ripening effects: the resulting pore size distribution is bidisperse as well, i.e. it highlights two monodisperse foam distributions, both of them being characterized by an average pore size value is equal to initial bubble size value within the experimental error bar. Ripening is however responsible for noticeable broadening of the respective monodisperse distributions. Such a broadening effect was found to be more pronounced for samples characterized by a large ratio $\left(D_{b 2}-D_{b 1}\right) / D_{b 1}$, but in all cases the relative spreading of each pore size distribution in bidisperse coatings is characterized by a value $\Delta D_{p} / D_{p}$ smaller than $15 \%$. Samples presented in Figure 2 have $80 \% / 20 \%$ of small/large pores (in number) but the method has been successfully used to produce coatings with other proportions, i.e. $20 \% / 80 \%$ and $50 \% / 50 \%$.

As expected, coatings made with polydisperse precursor foam have polydisperse pore sizes (see Figure $2 \mathrm{~h}$-j). In agreement with literature [11], [20], the measured pore sizes are well described by a log-normal distribution curve. All the measured pore size distributions showed the same log-normal behavior in our polydisperse coatings.

In the following we try to justify the fact that bubble size distributions are only weakly modified by foam aging mechanisms during after foam shaping. The characteristic time $t_{r}$ for foam ripening will be compared with the time required to solidify the fresh coating. The characteristic time $t_{r}$ for foam ripening can be expressed as follows [11]: $t_{r} \approx D_{b}^{2} / 2 K_{2}$, where $K_{2}$ has dimension of a diffusion coefficient with order of magnitude around $40.10^{-12} \mathrm{~m}^{2} \cdot \mathrm{s}^{-1}$ [21] for the type of surfactant used in our study and for gas volume fraction around 0.8. Thus, following characteristic times are calculated: $t_{r} \approx 1500 \mathrm{~s}$ for $D_{b}=350 \mu \mathrm{m}$, and $t_{r} \approx 300 \mathrm{~s}$ for $D_{b}=150 \mu \mathrm{m}$. Those times should be compared to the time $t_{j}$ required to solidify the fresh PUD foam. Observation of the coatings' surface during drying has shown that the samples didn't evolve anymore after typically 30 min. This order of magnitude can be understood as follows: First, solidification is assumed to occur due to water evaporation, as the volume fraction of the bubble-embedded PUD (i.e. $\varphi_{P U D}$ ) reaches the jamming volume fraction $\varphi_{P U D}^{j}$. The latter can be understood as the packing volume fraction for the PUD particles, i.e. $\varphi_{P U D}^{j} \approx 0.7$. Then the water evaporation rate can be expressed as [22] $d V_{w} / d t=2 D_{m} L\left(\rho_{\text {sat }}-\rho_{\infty}\right) / \rho$, where $\rho_{\text {sat }}$ is the density of water vapor at saturation in air, $\rho_{\infty}=R H \rho_{\text {sat }}$ corresponds to the value in the drying atmosphere, $D_{m}$ is the diffusion coefficient for water molecules in air, and $L \approx 0.01 \mathrm{~m}$ is the size that characterizes the sample's surface area (width). Using values $D_{m} \approx 0.3 \mathrm{~cm}^{2} \cdot \mathrm{s}^{-1}, \rho_{\text {sat }}=0.02 \mathrm{~kg} \cdot \mathrm{m}^{-3}$ and $\mathrm{RH}=0.4$, we obtain $d V_{w} / d t \approx 210^{-11} \mathrm{~m}^{3} \cdot \mathrm{s}^{-1}$. For a sample's volume equal to $L^{2} e$, the corresponding water volume is $V_{w}=\left(1-\varphi_{P U D}\right)(1-\phi) L^{2} e$. The volume of water to be evaporated for reaching the jammed state (j) from the initial state (i) of the fresh coating is $\Delta V_{w}=V_{w}^{i}-V_{w}^{j}$ and the time for solidification is 
$t_{j}=\Delta V_{w} /\left(d V_{w} / d t\right)$. According to the production parameters, the solidification time is obtained: $t_{j} \approx 1000 s$.

Now we can compare the value $t_{j}$ with values obtained for the ripening time $t_{r}$ : for $D_{b}>300 \mu \mathrm{m}$ we obtain $t_{r}>t_{j}(1500 \mathrm{~s}$ vs $1000 \mathrm{~s})$, which means that such bubbles are not expected to ripen significantly before the foam solidifies. On the other hand, foam made with smaller bubbles can be impacted by ripening: for $D_{b}=150 \mu \mathrm{m}, t_{r} \approx 300 \mathrm{~s}$. This prediction is consistent with our observations and this sets the limits of the method with such systems: morphology control with small bubbles requires to slowdown the ripening process (see [21] for more details) or to accelerate the solidification time.

\subsection{Control of the coating's density}

For a given sample to be produced, the density can be targeted by considering the quantity of PU introduced in the foam during the mixing step. Using the production parameters, the volume of PU introduced in the foam coating during time $\Delta t$ is $\varphi_{P U D}^{0} Q_{P U D} \Delta t$, whereas the produced volume of fresh PUD foam is $\left(Q_{P U D}+Q_{p f}^{*}\right) \Delta t$. The latter decreases due to water evaporation during the drying step and reaches the final value $\left(\varphi_{P U D}^{0} Q_{P U D}+\phi_{0} Q_{p f}^{*}\right) \Delta t$. Therefore, the final solid (PUD) volume fraction can be estimated by the following relation:

$$
\phi_{s}=\frac{\varphi_{P U D}^{0} \times Q_{P U D}}{\varphi_{P U D}^{0} \times Q_{P U D}+\phi_{0} \times Q_{p f}^{*}}=\frac{1}{1+\left(\frac{\phi_{0}}{\varphi_{P U D}^{0}}\right)\left(\frac{Q_{p f}}{Q_{P U D}}+c\right)} \quad \text { (eq.1) }
$$

where the numerical coefficient $c$ depends on the setup and should be considered as a fitting parameter. Figure 3 shows the results for solid fraction $\phi_{s}$ measured for more than 60 samples within the range 0.09-0.26. Equation 1 shows very good agreement over the full range of investigated flow rates using the coefficient $c=0.4$. As discussed in section 2.2.2, this value means that excess pressure in the foam column increases as a function of $Q_{P U D}$ (or equivalently as a function of $Q_{P U D} / Q_{p f}$ ) up to 0.3 bar for $Q_{P U D} / Q_{p f} \approx 0.8$. Figure 3 demonstrates that the solid volume fraction $\phi_{S}$, as well as the coating density, can be easily adjusted by varying the production parameters. 


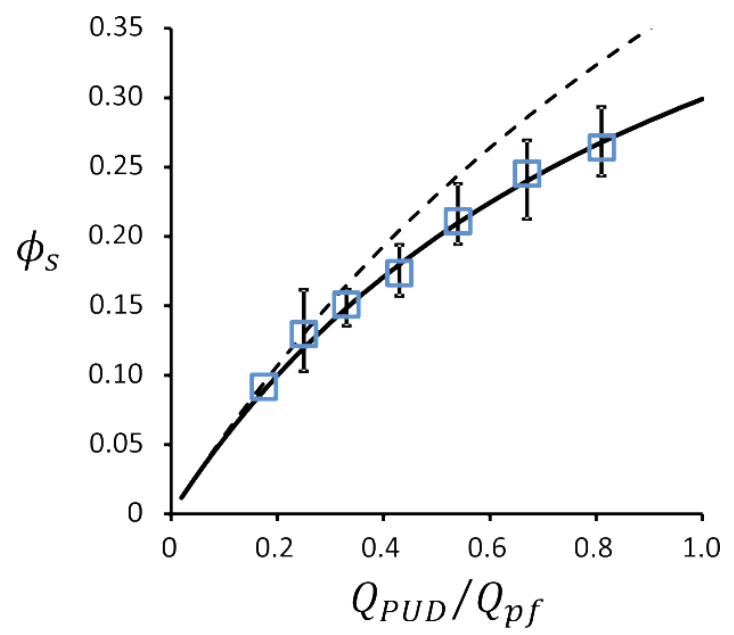

Figure 3: Solid volume fraction measured for more than 60 foam coatings as a function of the ratio of PUD solution and precursor foam during the production process (see Figure 1). The dashed line corresponds to equation 1 with $\phi_{0}=0.92, \varphi_{P U D}^{0}=0.55$ and $\mathrm{c}=0$. The solid line corresponds to equation 1 with $\phi_{0}=0.92, \varphi_{P U D}^{0}=0.55$ and $\mathrm{c}=0.4$.

Structural characteristics of the samples are presented in Figure 4 showing the solid fraction $\phi_{S}(z)$ obtained from tomography slices as a function of vertical position $z$ across the coating's thickness for typical monodisperse (Figures 4a,b), bidisperse (Figure 4e) and polydisperse (Figure 4f) samples. Each type of sample appears to be homogeneous, with $\phi_{S}(z)$ oscillating around the average solid volume fraction $\left\langle\phi_{s}\right\rangle$. We will discuss further in the next paragraph the pattern observed for $\phi_{S}(z)$ in monodisperse coatings. The absence of any gradient for solid volume fraction across the thickness means that the effects of drainage, i.e. bubble rising and downwards liquid flow, have been very weak before solidification. Let us estimate the expected deviation for gas volume fraction between top and bottom for a fully drained aqueous foam (or equivalently the corresponding solidified foam) of thickness $e=1200 \mu \mathrm{m}[23]: \phi_{s}^{-1 / 2}(z) \cong \phi_{s}^{-1 / 2}(0)+\sqrt{3} \rho g D_{b} z / 2 \gamma$, where $\rho$ is the density of the liquid phase. In assuming that $\phi_{s}(0)=0.26[12]$, we find that $\phi_{s}(e)=0.23$ for $D_{b}=150 \mu \mathrm{m}, \phi_{s}(e)=0.20$ for $D_{b}=$ $600 \mu \mathrm{m}$, and $\phi_{s}(e)=0.17$ for $D_{b}=1200 \mu \mathrm{m}$. With respect to the average value $\left\langle\phi_{s}\right\rangle$ of the theoretical profile, this corresponds to relative deviations $\left(\phi_{S}(0)-\phi_{S}(e)\right) /\left\langle\phi_{S}\right\rangle$ equal respectively to $15 \%, 30 \%$ and 45\%. Those values are larger than observed deviations, which seems to indicate that the effect of drainage is limited by kinetics. The characteristic drainage time is $t_{D} \sim e / v_{D}$, where $v_{D}=k\left(\phi, D_{b}\right) \rho g / \mu$ is drainage velocity (Darcy) with $k\left(\phi, D_{b}\right)$ the foam permeability ( $\phi$ is the gas volume fraction of the fresh PUD foam) and $\mu$ the shear viscosity of the PUD [11]. For $\phi \approx 0.75, k / D_{b}{ }^{2} \approx 10^{-3}$ [24], and using $500 \mathrm{mPa}$.s as an initial value for $\mu$, we obtain: $t_{D} \approx 10 \mathrm{~h}$ for $D_{b}=150 \mu \mathrm{m}, 2 \mathrm{~h}$ for $D_{b}=600 \mu \mathrm{m}$ and $50 \mathrm{~min}$ for $D_{b}=1200 \mu \mathrm{m}$. Therefore, most of the foam coatings are characterized by $t_{D}>t_{j}$, i.e. the 
coatings are expected to solidify before drainage has time to occur significantly. This prediction is indeed consistent with our observations.
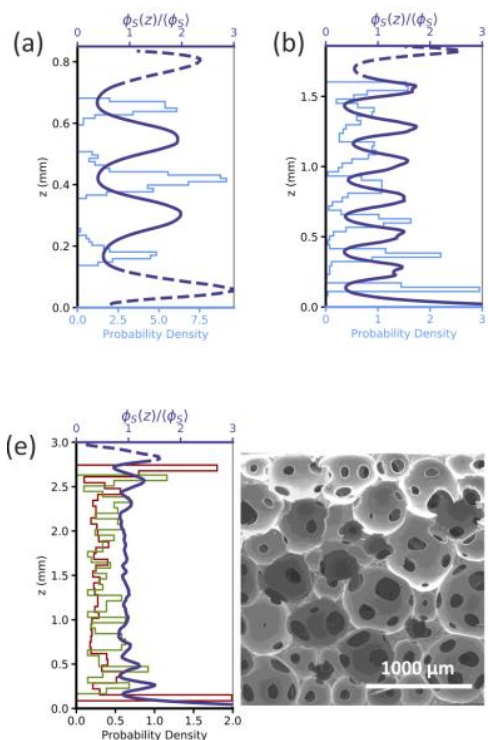

(c)

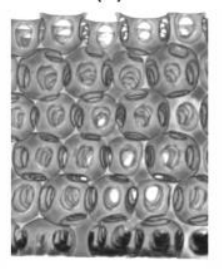

(f)

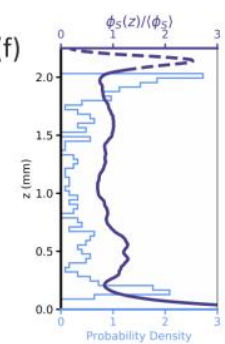

(d)

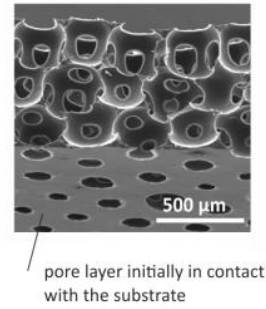

with the substrate

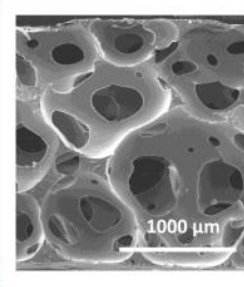

Figure 4: $(a, b, e, f)$ Typical solid volume fraction profile $\phi_{s}(z)$ and pore position probability density (number weighted) measured across the coating's thickness e. Dashed curves indicate areas for which solid fraction should be considered as an "apparent" value due to the "roughness" at the free top surface of the samples. A typical SEM side view is showed for cases (e) and (f). (a) Monodisperse sample with three pore layers: $e=830 \mu \mathrm{m},\left\langle D_{p}\right\rangle=$ $330 \mu \mathrm{m},\left\langle\phi_{s}\right\rangle=0.14$. (b) Monodisperse sample with seven pore layers: $\left.\mathrm{e}=1860 \mu \mathrm{m},\left\langle\mathrm{D}_{\mathrm{p}}\right\rangle=330 \mu \mathrm{m},\left\langle\phi_{s}\right\rangle=0.16\right)$. (c) Tomographic image of a monodisperse sample with six pore layers: $\mathrm{e}=1600 \mu \mathrm{m},\left\langle\mathrm{D}_{\mathrm{p}}\right\rangle=330 \mu \mathrm{m},\left\langle\phi_{s}\right\rangle=0.14$. (d) show the pore contact layer (bottom) of a coating that has been removed from the substrate, revealing pore apertures. (e) Bidisperse sample: $\mathrm{e}=3000 \mu \mathrm{m},\left\langle D_{p_{1}}\right\rangle=290 \mu \mathrm{m}$ (brown), $\left\langle D_{p_{2}}\right\rangle=550 \mu \mathrm{m}$ (green), $\left\langle\phi_{s}\right\rangle=0.13$, number proportions of small/large pores: $80 \% / 20 \%$. (f) Polydisperse sample: $\mathrm{e}=2250 \mu \mathrm{m},\left\langle\mathrm{D}_{\mathrm{p}}\right\rangle=350 \mu \mathrm{m}$,

$$
\left\langle\phi_{\mathrm{s}}\right\rangle=0.13 \text {. }
$$

\subsection{Control of structural order within the coatings}

Structural orders observed within the coatings are discussed, firstly with the monodisperse coatings. The free-surface of those coatings exhibits a triangular arrangement of the pores (see Figure 2a) and the bulk has a layered structure, as revealed by the side-view image of figures $4 c, d$. The bulk structure is described more quantitatively by the vertical distribution of pore centers or, equivalently, by the "oscillation" pattern of the density profiles (see Figure 4a,b). Therefore, for monodisperse foams, the number of minima is equal to the number $n_{\ell}$ of pore layers across the coating's thickness. Those structural features, which are induced by the plane boundary at bottom, have been observed for coatings up to seven layers. 
Moreover, the coatings are fully open-cell foams, with the upper pores open at the coating's surface (see Figures $2 \mathrm{~b}$ and $4 \mathrm{c}, \mathrm{d})$. The lack of membrane between the pores can be understood by considering that the micrometer-sized PU particles are squeezed out of bubble contact areas before and during the drying process. This effect can be observed also for the pore layer initially in contact with the substrate (see Fig. 4d). Note however that removal of the coating was allowed without damage only with silicone-coated substrates, so it is hard to draw a general conclusion about this issue. The resulting coating's structure is a network of struts with apertures whose geometrical features are important for several properties of the coating. For example, the strut thickness controls the mechanics [3], whereas apertures control the foam permeability for fluid flows [25] and for acoustics [26]. Interestingly, the aperture diameter $d_{a}$ can be fully controlled through bubble size and solid volume fraction [11], i.e. $d_{a} / D_{b}=f\left(\phi_{s}\right)$, as demonstrated in Figure 5. The apertures of the upper layer are supposed to ensure connection of the bulk porosity with environment and they are therefore of prime interest. In Figure 5 we show that the surface fraction $\Psi$ occupied by the circular holes at the surface (see figure $2 b$ for example where $\Psi \approx$ 0.2) decreases but not vanishes as a function of $\phi_{s}$, which means that the coating's porosity would be always reachable for subsequent treatment. Note that (1) $\Psi$ is not equal to the fraction of foam films covering a wall in contact with aqueous foam [27], i.e. $\Psi=\left(1-\sqrt{\phi_{S} / 0.36}\right)^{2}$, which suggests that the holes opening process is complex, and (2) such surface texture is known to impact the wetting properties of surfaces, as described by the Cassie-Baxter model [1].

$\mathrm{Bi}$-disperse coatings exhibit less order than monodisperse ones (see figure $2 \mathrm{~d}$-f for the upper pore layer, and figure $4 \mathrm{e}$ for the bulk), and the layered structure is completely lost when making polydisperse coatings, where some bubbles have size comparable to the coating's thickness (see figure $4 \mathrm{f}$ ). Apertures both at surface and in bulk of bidisperse coatings exhibit a significant range of sizes, which means that mixing two monodisperse bubble assemblies is sufficient for opposing the ordering effect induced by the plane boundary at bottom. Note that the values chosen for both the bubble size ratio and mixture proportions in bidisperse samples are close to values corresponding to the local minimum observed for the critical volume fraction of 2D bidisperse particle assemblies [28]. Different values for those parameters may not be so efficient for opposing the ordering effect of the wall. Note also that bidisperse and polydisperse coatings exhibit smaller values for $\Psi$ than for monodisperse ones (see figure 5). This result is consistent with observations made at wall contacting aqueous foam [27]. 


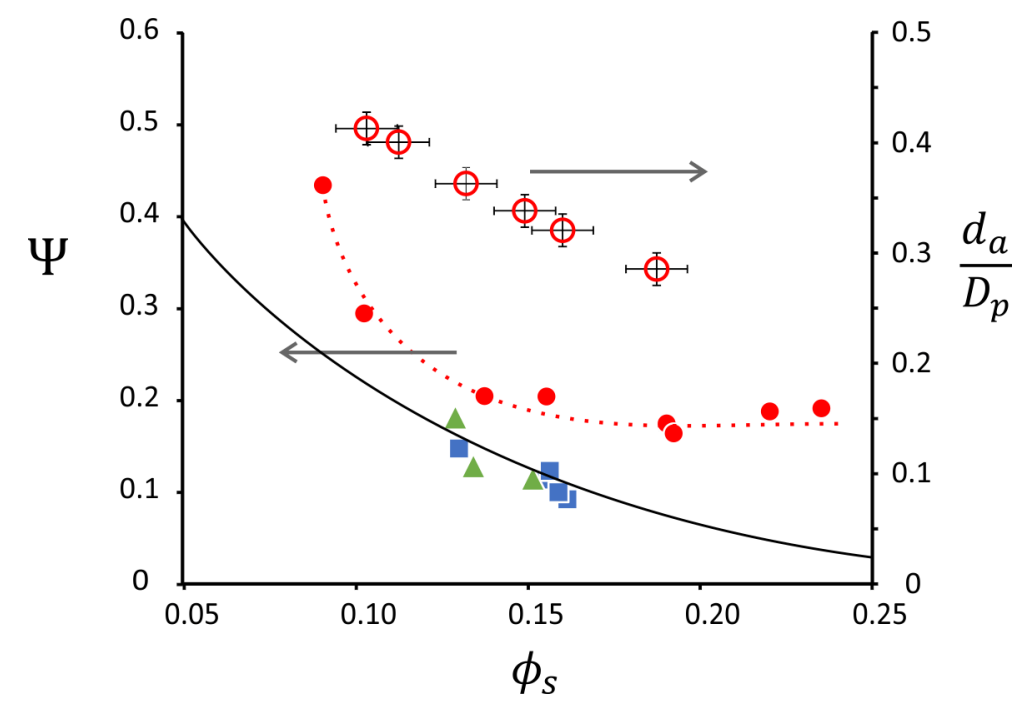

Figure 5: Apertures in the foam coatings. Surface fraction $\Psi$ of holes at the surface (full symbols, circles: monodisperse, squares: bi-disperse, and triangle: polydisperse), and ratio of average diameter $d_{a}$ of apertures between bulk pores divided by pore diameter $D_{p}$ (empty symbols, monodisperse). Samples correspond to pore distributions shown in Figures $2 c, g$, j. The dotted line is a guide for the eye. The solid line corresponds to the equation $\Psi=\left(1-\sqrt{\phi_{s} / 0.36}\right)^{2}$.

\subsection{Control of the coating's thickness}

Results for the variation of coatings' thickness are presented in Figure 6 as a function of spreading velocity. For monodisperse coatings, the foam bubbles were found to organize spontaneously as stacks of bubble layers with triangular order. In that case, the spreading velocity $v$ can be used as an efficient way for tuning the coating's thickness $e$, or equivalently the number of layers $n_{\ell}$ (see Figure 6a). This result can be understood by considering the volume conservation for the PUD foam during the spreading process, which can be written:

$$
v\left(n_{\ell}\right)=C \frac{Q_{p f}^{*}+Q_{P U D}}{w \times D_{b} \times n_{\ell}} \quad(\text { eq.2) }
$$

where $w$ is the width of the nozzle and $C$ is a numerical coefficient (which accounts for example for shrinkage during drying). Equation 2 is fitted to experimental data (obtained for $D_{b}$ (or $D_{p}$ ) $=330 \mu \mathrm{m}$ and $\left.\phi_{s}=0.14\right)$ in Figure $6 a$, showing good agreement with $C \approx 1.5$. All the monodisperse coatings were found to be described using equation 2 , and the resulting coating's thickness is given by $e \approx 0.8 \times n_{l} \times$ $D_{p}$ (see inset in figure 6a). The coefficient 0.8 is probably reminiscent of hard spheres close packings 
observed for those samples. Note that (1) choosing $v$ values that are intermediate between $v\left(n_{\ell}\right)$ and $v\left(n_{\ell}+1\right)$ results in the production of coatings counting alternatively $n_{\ell}$ and $n_{\ell}+1$ pore layers, and (2) although the nozzle's height $h$ does not set the coating's thickness, we observed that it has however to be chosen such that $0.5<\frac{h}{n_{\ell} D_{b}}<2$. Bidisperse and polydisperse foams also show a decrease of the coating thickness as a function of the spreading velocity (see Figure 6b). For such cases, the coating thickness is reasonably described by $C^{\prime}\left(Q_{p f}^{*}+Q_{P U D}\right) /(v \times w)$, with $C^{\prime} \approx 1.7$.
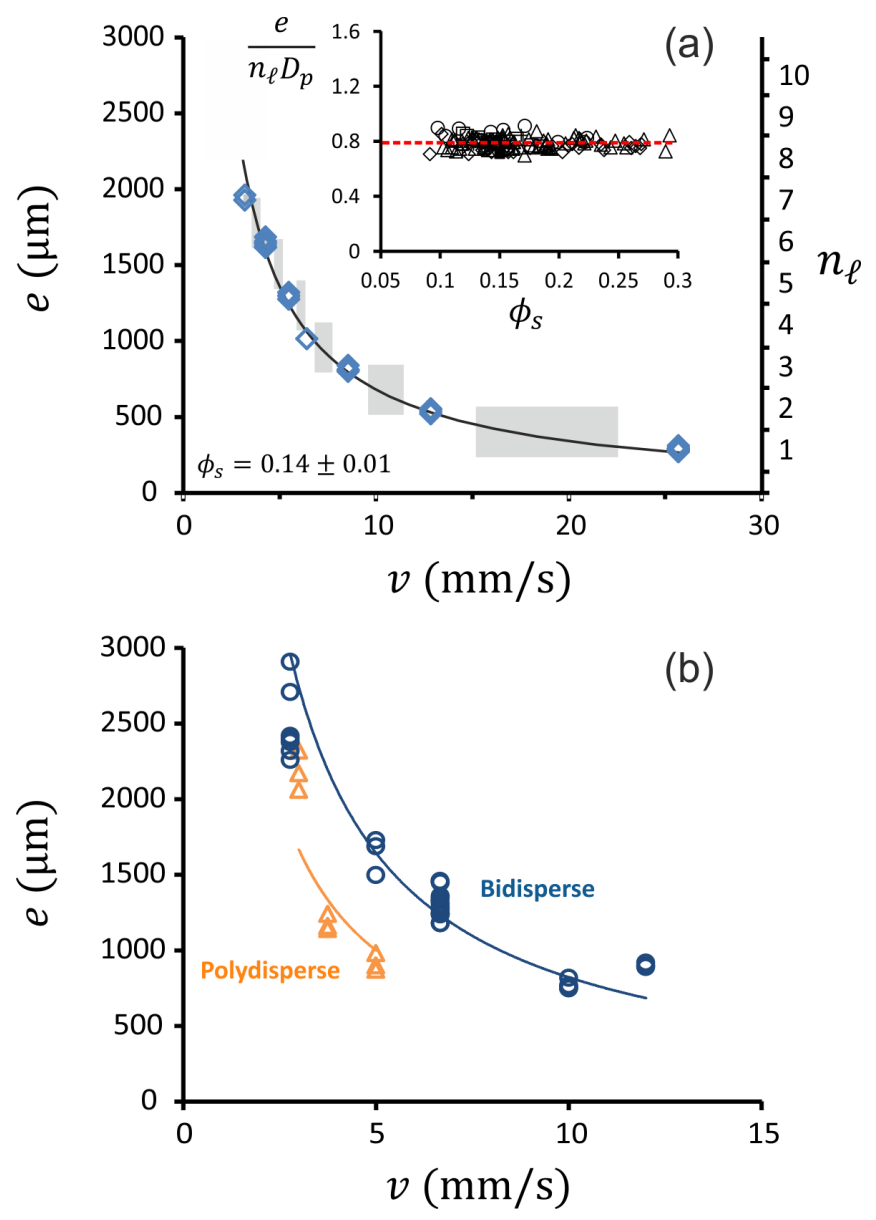

Figure 6: (a) Evolution of the measured thickness $e$ and the number of bubble layers $n_{\ell}$ in monodisperse coatings as a function of the spreading velocity $v$. The solid volume fraction is $\phi_{s}=0.14 \pm 0.01$ and the pore diameter is $D_{p}=330 \mu \mathrm{m}$. Nozzle's height used to spread the foam was: $h=0.3 \mathrm{~mm}$ for $n_{\ell}=1, h=1.2 \mathrm{~mm}$ for $n_{\ell}=2-6, h=$ $1.5 \mathrm{~mm}$ for $n_{\ell}=7$. The line corresponds to equation 2 with $C=1.5$. Grey areas correspond to velocity ranges for which the resulting coatings have not a constant thickness (i.e. mixtures of $n_{\ell}$ and $n_{\ell}+1$ layers). Inset: Coating's thickness divided by $n_{\ell} D_{p}$ as a function of $\phi_{s}$. (b) Same results for bidisperse (number proportions of $320 \mu \mathrm{m} / 540$ $\mu \mathrm{m}$ pores: $80 \% / 20 \%)$ and polydisperse foams. Lines correspond to equation $\mathrm{C}^{\prime}\left(\mathrm{Q}_{\mathrm{pf}}^{*}+\mathrm{Q}_{\mathrm{PUD}}\right) /(\mathrm{v} \times \mathrm{w})$, with $\mathrm{C}^{\prime} \approx 1.7$. 


\section{Conclusion}

A simple and efficient method has been presented for producing foam-based solid coatings. The coating is obtained by mixing separately-prepared aqueous precursor foam with particle suspension (here micrometer-sized polyurethane dispersion), and by spreading with a dedicated nozzle the resulting PUD foam on the surface to be coated. Further drying step allows for the fresh foam coating to be solidified while keeping the main features of the morphology that has been set during the spreading step. Due to their micrometer size, the polymer particles are squeezed out of the contact areas between the bubbles in the fresh foam, and therefore open-cell solid foams were obtained after the drying step.

The coating's thickness can be controlled accurately by tuning the spreading velocity. Pore size can be controlled through bubble size from the precursor aqueous foam. For the purpose of demonstrating that bubble size distribution of the precursor foam is preserved in the final solid coating, controlled monodisperse, bidiperse (i.e. mixture of two sizes) and polydisperse foam coatings were produced. Such a result has been obtained for several solid volume fractions within a wide range (0.080.3). Monodisperse samples were found to consist of stacks of ordered pore layers with a well-defined thickness. We show that this spontaneous ordering is destroyed when making bimodal distribution of comparable bubble sizes.

The proposed method was shown to allow parameters of microstructural elements to be controlled easily by tuning the bubble size of the precursor foam and the solid volume fraction of the coating. The surface porosity, which is supposed to be a crucial parameter for impregnation purpose or for tuning liquid wetting properties, is shown to be controlled as well. The resulting porous structure allows for various effective surface treatments, which increases drastically the spectrum of properties which can be achieved by using foam-based coatings, i.e. superhydrophobicity [29], [30], antibacterial [31], electrical [32] or fire-safe [33] properties.

Coatings on silicone-treated substrates were allowed to be removed without damage, revealing apertures in the contact pore layer and suggesting that the adhesion energy of such foam coatings is modulated by the fraction of matrix in true contact with the substrate. However, a general conclusion about this issue cannot be drawn from the present work, so a dedicated study of the foam/substrate properties should be performed.

Results obtained with the studied polyurethane dispersion are expected to be reproduced with other dispersions. Note that the precursor does not need to be so controlled as we did for the present 
study, which means that significantly larger flow rates of precursor foam could be achieved and that such a coating method could be developed at larger scale if required.

\section{Acknowledgements}

We gratefully acknowledge financial support from Agence Nationale de la Recherche (Grant no. ANR-13RMNP-0003-01).

\section{References}

[1] A. B. D. Cassie and S. Baxter, "Wettability of porous surfaces," Trans. Faraday. Soc., no. 5, pp. 546-551, 1944.

[2] N. J. Glassmaker, A. Jagota, C. Hui, W. L. Noderer, and M. K. Chaudhury, "Enhanced Adhesion," Proceedings of the National Academy of Sciences of the United States of America, vol. 104, no. 26, pp. 1-6, 2007.

[3] L. J. Gibson and M. F. Ashby, Cellular Solids: Structure and Properties. Cambridge: Cambridge University Press, 1997.

[4] B. François, O. Pitois, and J. François, "Polymer films with a self-organized honeycomb morphology," Advanced Materials, vol. 7, no. 12, 1995.

[5] S. Andrieux, A. Quell, C. Stubenrauch, and W. Drenckhan, "Liquid foam templating - A route to tailor-made polymer foams," Advances in Colloid and Interface Science, vol. 256, pp. 276-290, 2018.

[6] S. Andrieux, W. Drenckhan, and C. Stubenrauch, "Highly ordered biobased scaffolds: From liquid to solid foams," Polymer, vol. 126, pp. 425-431, 2017.

[7] A. Testouri, C. Honorez, A. Barillec, D. Langevin, and W. Drenckhan, "Highly structured foams from chitosan gels," Macromolecules, vol. 43, no. 14, pp. 6166-6173, 2010.

[8] M. Costantini, J. Jaroszewicz, L. Kozon, karol Szlazak, W. Swieszkowski, P. Garstecki, C. Stubenrauch, A. Barbetta, and J. Guzowski, "3D Printing of Functionally Graded Porous Materials Using On-Demand Reconfigurable Microfluidics," Angewandte Chemie, p. ange.201900530, 2019.

[9] C. Poulard, S. Levannier, A. Gryson, M. Ranft, and W. Drenckhan, "Structuring Solid Surfaces with Bubble Coatings," Advanced Engineering Materials, vol. 19, no. 2, pp. 1-7, 2017.

[10] D. Dieterich, "Aqueous Emulsions, Dispersions and Solutions of Polyurethanes; Synthesis and Properties," Prog. Org. Coat., vol. 9, pp. 281-340, 1981.

[11] I. Cantat, S. Cohen-Addad, F. Elias, F. Graner, R. Höhler, O. Pitois, and F. Rouyer, Foams: Structure and Dynamics. Oxford: Oxford University Press, 2013.

[12] F. Gorlier, Y. Khidas, and O. Pitois, "Coupled elasticity in soft solid foams," Journal of Colloid and Interface Science, vol. 501, pp. 103-111, 2017. 
[13] B. Haffner, Y. Khidas, and O. Pitois, "Flow and jamming of granular suspensions in foams.," Soft matter, vol. 10, no. 18, pp. 3277-3283, 2014.

[14] Y. Khidas, B. Haffner, and O. Pitois, "Critical size effect of particles reinforcing foamed composite materials," Composites Science and Technology, vol. 119, pp. 62-67, 2015.

[15] F. Gorlier, Y. Khidas, and O. Pitois, "Elasticity of particle-loaded liquid foams," Soft Matter, vol. 13, no. 25, pp. 4533-4540, 2017.

[16] J. Schindelin, I. Arganda-Carreras, E. Frise, V. Kaynig, M. Longair, T. Pietzsch, S. Preibisch, C. Rueden, S. Saalfeld, B. Schmid, J.-Y. Tinevez, D. J. White, V. Hartenstein, K. Eliceiri, P. Tomancak, and A. Cardona, "Fiji: an open-source platform for biological-image analysis," Nature Methods, vol. 9, no. 7, pp. 676-682, Jul. 2012.

[17] C. T. Rueden, J. Schindelin, M. C. Hiner, B. E. DeZonia, A. E. Walter, E. T. Arena, and K. W. Eliceiri, "ImageJ2: ImageJ for the next generation of scientific image data," BMC Bioinformatics, vol. 18, no. 1, p. 529, Dec. 2017.

[18] D. Legland, I. Arganda-Carreras, and P. Andrey, "MorphoLibJ: integrated library and plugins for mathematical morphology with ImageJ," Bioinformatics, vol. 32, no. 22, pp. 3532-3534, Jul. 2016.

[19] N. Otsu, "A Threshold Selection Method from Gray-Level Histograms," IEEE Transactions on Systems, Man, and Cybernetics, vol. 9, no. 1, pp. 62-66, Jan. 1979.

[20] J. Lambert, R. Mokso, I. Cantat, P. Cloetens, J. a. Glazier, F. Graner, and R. Delannay, "Coarsening foams robustly reach a self-similar trowth regime," Physical Review Letters, vol. 104, no. 24, pp. 1-4, 2010.

[21] O. Pitois, Foam ripening, in Foam Engineering: Fundamentals and Applications, John Wiley. John Wiley \& Sons, Ltd., 2012.

[22] A. M. Cazabat and G. Guéna, "Evaporation of macroscopic sessile droplets," Soft Matter, vol. 6, no. 12, pp. 2591-2612, 2010.

[23] A. Maestro, W. Drenckhan, E. Rio, and R. Höhler, "Liquid dispersions under gravity: volume fraction profile and osmotic pressure," Soft Matter, vol. 9, no. 8, p. 2531, 2013.

[24] F. Rouyer, O. Pitois, E. Lorenceau, and N. Louvet, "Permeability of a bubble assembly: From the very dry to the wet limit," Physics of Fluids, vol. 22, no. 4, 2010.

[25] V. Langlois, V. H. Trinh, C. Lusso, C. Perrot, X. Chateau, Y. Khidas, and O. Pitois, "Permeability of solid foam: Effect of pore connections," Physical Review E, vol. 97, no. 5, 2018.

[26] V. H. Trinh, M. T. Hoang, C. Perrot, V. Langlois, Y. Khidas, and O. Pitois, "A Systematic Link between Microstructure and Acoustic Properties of Foams: A Detailed Study on the Effect of Membranes," in Poromechanics 2017 - Proceedings of the 6th Biot Conference on Poromechanics, 2017.

[27] E. Forel, E. Rio, M. Schneider, S. Beguin, D. Weaire, S. Hutzler, and W. Drenckhan, "The surface tells it all: relationship between volume and surface fraction of liquid dispersions," Soft Matter, vol. 12, no. 38, pp. 8025-8029, Sep. 2016. 
[28] D. J. Koeze, D. Vågberg, B. B. T. Tjoa, and B. P. Tighe, "Mapping the jamming transition of bidisperse mixtures," EPL (Europhysics Letters), vol. 113, no. 5, p. 54001, Mar. 2016.

[29] Z. Zhang, H. Wang, Y. Liang, X. Li, L. Ren, and Z. Cui, “One-step fabrication of robust superhydrophobic and superoleophilic surfaces with self- cleaning and oil / water separation function," Scientific Reports, vol. 8:3869, pp. 1-12, 2018.

[30] X. Gao, J. Zhou, R. Du, Z. Xie, S. Deng, and R. Liu, "Robust Superhydrophobic Foam : A GraphdiyneBased Hierarchical Architecture for Oil / Water Separation," Advanced Materials, vol. 28, no. 1, pp. 168-173, 2015.

[31] P. Jain and T. Pradeep, "Potential of Silver Nanoparticle-Coated Polyurethane Foam As an Antibacterial Water Filter," Biotechnology and Bioengineering, vol. 90, no. 1, pp. 59-63, 2005.

[32] X. Xinzhao, L. Guoming, L. Dongyan, S. Guoxin, and Y. Rui, "Electrically conductive graphenecoated polyurethane foam and its epoxy composites," Composites Communications, vol. 7, pp. 16, 2018.

[33] H. Xie, X. Lai, Y. Wang, H. Li, and X. Zeng, "A green approach to fabricating nacre-inspired nanocoating for super-efficiently fire-safe polymers via one-step self-assembly," Journal of Hazardous Materials, vol. 365, pp. 125-136, 2019. 